Exam nat i on of val i di ty of $f$ al I risk assessment items for screening hi gh fal I risk el derly anong the heal thy communi ty-dwel I i ng Japanese popul at i on

\begin{tabular}{|c|c|}
\hline 著者 & $\begin{array}{l}\text { DEMURA Shi ni chi, SATO Susume, YAMAI Shunsuke, } \\
\text { KASUGA Kosho, NAGASAMA Yoshi nor i }\end{array}$ \\
\hline $\begin{array}{l}\text { jour nal or } \\
\text { publ i cat i on titl e }\end{array}$ & Ar chi ves of Ger ont ol ogy and Ger i at ri cs \\
\hline vol une & AGG 2356 \\
\hline page $r$ ange & $1-5$ \\
\hline year & 2010 \\
\hline URL & ht t p: //hdl . handl e. net /10098/2976 \\
\hline
\end{tabular}




\title{
Examination of validity of fall risk assessment items for screening high fall risk elderly among the healthy community-dwelling Japanese population
}

\author{
Shinichi Demura ${ }^{a}$, Susumu Sato ${ }^{\text {b,* }}$, Shunsuke Yamaji ${ }^{c}$, Kosho Kasuga ${ }^{d}$, Yoshinori Nagasawa ${ }^{e}$ \\ ${ }^{a}$ Graduate School of Natural Science and Technology, Kanazawa University, Kakuma, Kanazawa, Ishikawa 920-1192, Japan \\ ${ }^{\mathrm{b}}$ Life-long Sports Core, Kanazawa Institute of Technology, Ohgigaoka 7-1, Nonoichi, Ishikawa 921-8501, Japan \\ ${ }^{\text {c } F a c u l t y ~ o f ~ M e d i c a l ~ S c i e n c e, ~ U n i v e r s i t y ~ o f ~ F u k u i, ~ S h i m o a i t s u k i ~ 23-3, ~ M a t s u o k a, ~ E i h e i j i, ~ Y o s h i d a, ~ F u k u i ~ 910-1193, ~ J a p a n ~}$ \\ ${ }^{\mathrm{d}}$ Faculty of Education, Gifu University, Yanagido 1-1, Gifu 501-1193, Japan \\ ${ }^{\mathrm{e}}$ Health and Sports Sciences, Kyoto Pharmaceutical University, Nakauchicho 5, Misasagi, Yamashinaku, Kyoto, 607-8414, Japan
}

\section{A R T I C L E I N F O}

\section{Article history:}

Received 10 May 2010

Received in revised form 7 October 2010

Accepted 9 October 2010

\section{Keywords:}

Accidental falls of elderly

Discriminant analysis

Cross-sectional study

\begin{abstract}
A B S T R A C T
We aimed to examine the validity of fall risk assessment items for the healthy community-dwelling elderly Japanese population. Participants were 1122 healthy elderly individuals aged 60 years and over (380 males and 742 females). The percentage who had experienced a fall was $15.8 \%$. This study used fall experience and 50 fall risk assessment items representing the five risk factors (symptoms of falling, physical function, disease and physical symptom, environment, and behavior and character), as we described before. The accuracy of predicting fall experience from the total score or each risk factor score was examined by discriminant analysis. The percentage correctly distinguishing the faller from the total score was $14.4 \%$, and that from the five risk factor scores was $39.7 \%$. This percentage, when using each risk factor score as an independent variable, was $42.5 \%$ (symptom of falling), $0.6 \%$ (physical function score), $0.6 \%$ (disease and physical symptoms score), $0.0 \%$ (environment score), and $1.1 \%$ (behavior and character score), respectively. The best predictor of fall experience of the community-dwelling elderly was the "symptom of falling" score. For fall risk assessment of the community-dwelling elderly, both of screening of fall risk level and assessing risk profile comprehensively is important.
\end{abstract}

(c) 2010 Elsevier Ireland Ltd. All rights reserved.

\section{Introduction}

Falls are a major public health issue in the elderly population, and there has been a focus on fall prevention based on fall risk assessment (Tinetti et al., 1988; Graafmans et al., 1996; American Geriatrics Society, 2001; Perell et al., 2001; Chan et al., 2006; Russell et al., 2009). To prevent falls in the elderly, it is important that the outcome of a fall risk assessment to provide a comprehensive fall risk level and fall risk profile, which identifies any problems for individuals.

In Japan, the fall risk assessment chart, which has been developed by the Tokyo Metropolitan Institute of Gerontology (TMIG), is widely used for the healthy community-dwelling elderly population (Suzuki, 2000, 2003). This assessment chart has criteria for screening persons with high fall risk, but it has problems in its assessment of a fall risk profile. Thus, this chart is limited in identifying physical function problems of the healthy elderly population because it is composed of only a few items for assessment of physical function. For the healthy

\footnotetext{
* Corresponding author. Tel.: +81 76248 1100x2386; fax: +81 762946704

E-mail address: sssato@neptune.kanazawa-it.ac.jp (S. Sato).
}

elderly population, especially, identification of problems regarding physical function is important to assess fall risk and to develop countermeasures for the prevention of falls, though disease is also one important risk factor for falling. Furthermore, the TMIG assessment chart also has problems in assessing longitudinal fall risk. Among fall risk factors, there is little improvement with regard to chronic disease. Therefore, excessive emphasis on immutable risk factors may limit longitudinal assessment of fall risk in the healthy community-dwelling elderly population (Schenkman and Riegger-Krugh, 1997). A comprehensive and gradual assessment of physical function is important (Demura et al., 2010).

We have attempted to select useful 50 fall risk assessment items (Demura's fall risk assessment chart: DFRA) for healthy community dwelling elderly from 5 risk factors of "symptoms of falling", "physical function", "disease and physical symptoms", "environment", and "behavior and character" (Demura et al., 2010). The difference with the TMIG assessment chart is that our assessment items assumed "symptoms of falling" as a risk factor, and can comprehensively assess physical function characteristics and functional levels. This study examined the availability of these items for assessment of fall risk in the healthy communitydwelling elderly population. 
Fall risk refers to the possibility of falling in the future. The prospective studies regarding fall risk assessment confirm the actual incidence of falling after a few years. On the other hand, cross-sectional studies used fall experience as a criterion for assessment of validity, and it has been observed whether the assessment chart (assessment items) can predict fall experience. However, there are few reports regarding the validity of the TMIG assessment chart.

This study was based on cross-sectional data. We aimed to examine the validity (prediction accuracy of fall experience) of the DFRA.

\section{Participants and methods}

\subsection{Participants and data collection}

The participants were healthy and community-dwelling elderly individuals aged 60 and over, living in Akita, Kanagawa, Ishikawa, Fukui, Nagano, Gifu, Aichi, Tottori and Fukuoka Prefectures in Japan. Mail or field surveys were conducted for 1927 elderly subjects, and there were 1464 respondents. Among them, there were 1122 elderly (70.3 \pm 7.1 years) showing missing values of less than 10 percent and we turned them into an object of analysis. They were composed of 380 males ( $70.5 \pm 7.0$ years) and 742 females (70.4 \pm 7.2 years), and 177 of them (15.8\%) had a fall experience this past year.

\subsection{Fall risk assessment}

This study used the DFRA, which is composed of fall experience and 50 fall risk assessment items representing the five risk factors of "symptoms of falling," "physical function," "disease and physical symptoms," "environment," and "behavior and character," referring Demura et al. (2010). The symptoms of fall were assessed by 3 items (Fell like falling in the preceding year, stumble, and look like falling). The physical function was assessed by 22 items selected from three components (fundamental function, advanced function, and gait) and eight elements (muscular strength, lower limb strength, balancing ability, walking ability, going and down stairs, changing and holding posture, upper limb function, and gait). The diseases and physical symptoms were assessed by thirteen items selected from six components (dizziness and blackout, medication, sight/hearing and cognition disorder, cerebral vascular, arthritic and bone disease, and circulatory disease). The environment was assessed by four items selected from two components (surrounding environment, and clothing). The behavior and character were assessed by eight items selected from four components (inactivity, frequent urination, fear of falling, and risk behavior). All questions were responded to by dichotomous scale (yes or no), and the response with high risk category for each question was considered as a "high-risk response."

In addition, we also used a TMIG fall risk assessment chart (Suzuki, 2000, 2003). Also in fall risk assessment in the TMIG chart, all questions were responded to by dichotomous scale (yes or no), and the response with high risk category for each question was considered as a "high-risk response."

\subsection{Statistical analyses}

\subsubsection{Frequency distributions of total and risk factor scores of the DFRA}

The total score was calculated by summing up 50 item scores, and risk factor scores were calculated by summing items representing each risk factor. To confirm distribution characteristics of the total of the risk factor scores, relative frequency distribution (\%) and accumulative relative frequency distribution (\%) were calculated for both the faller and non-faller groups.

\subsubsection{Prediction of fall experience using total and risk factor scores of the DFRA}

This study examined the accuracy of predicting fall experience from each risk factor score and a total of those scores. Discriminant analysis, using fall experience as a dependent variable and the total score, which is based on 50 items, as an independent variable, was conducted and discriminate probability (percentages of distinguishing fallers and non-fallers correctly, respectively) was calculated. Then, a similar discriminant analysis using five risk factor scores as independent variables was conducted. Further, discriminant analysis using each risk factor score as an independent variable was conducted for each risk factor. To compare the prediction accuracy of the TMIG assessment chart, discriminant analysis was conducted using fall experience as a dependent variable and the total score as an independent variable. The total score of the TMIG assessment chart was calculated by summing up the scores of 14 items except for the item of "fall experience."

\subsubsection{Relationship between fall risk (fall experience), risk factor scores, and the total score}

Frequency for every total (risk factor) score was calculated for each faller and non-faller group, and then the odds ratio for each total (risk factor) score was calculated. In the case of "symptoms of falling," cross table based on fall experience ("faller" and "nonfaller") and risk factor score ("0 point" and "over 1 point") was made up and the odds ratio was calculated. Then, similar cross tables were made up for each risk factor score ("1 or more points" and "under 1 point," "2 or more points" and "under 2 points," and "3 points" and "under 3 points"), and odds ratios were calculated. Similar calculations were conducted for all risk factor scores and the total score.

Furthermore, frequency of fallers was calculated for every total and risk factor score, and the incidence of falling was calculated for every risk factor score and the total score.

\section{Results}

\subsection{Frequency distribution characteristics of total and each risk factor scores of the DFRA}

Fig. 1a shows the relative frequency distribution and accumulative relative frequency distribution in the total score for each faller and non-faller group. The distribution of the total score in the faller group shifted to the right side compared to non-faller group. The mean total score was significantly greater in the faller group (18.2 \pm 9.8 point) than in non-faller group ( $12.4 \pm 6.9$ point $)$.

Similarly, the relative frequency distribution and accumulative relative frequency distribution were calculated for every risk factor score (Fig. 1b-f). There were significant differences in all risk factor scores between faller and non-faller groups. The difference in these frequency distributions between faller and non-faller groups was found in "symptoms of falling." Although slightly different between the faller and non-faller groups, distinctive distribution characteristics were found in "physical function," "disease and physical symptoms," and "behavior and character," there was little difference based on "environment."

\subsection{Prediction of fall experience from total and risk factor scores of the DFRA}

Discriminant analyses were conducted using fall experience as a dependent variable and the total score or risk factor scores as independent variables. Table 1 shows the summary of percentages 

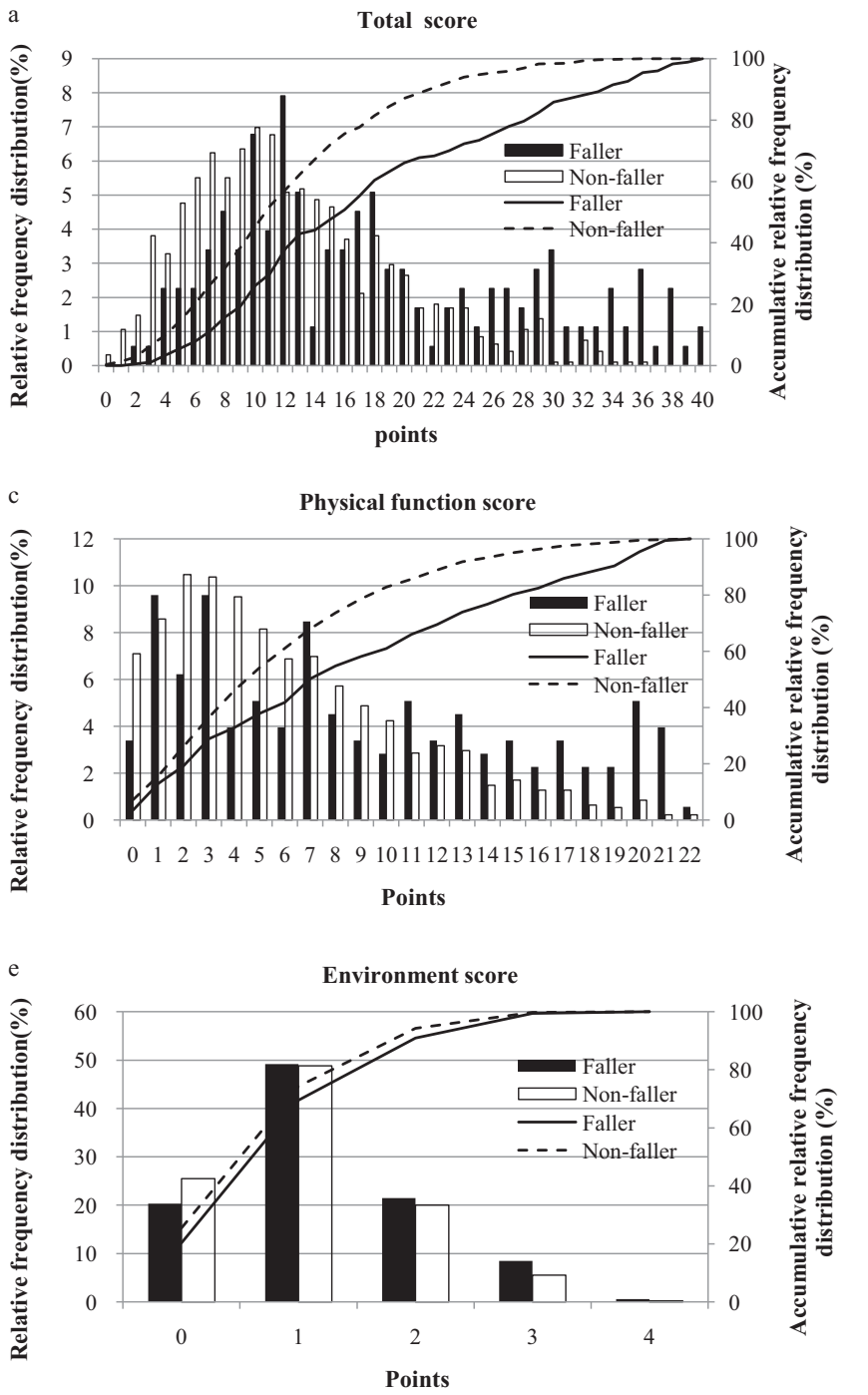

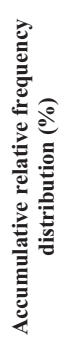

Points
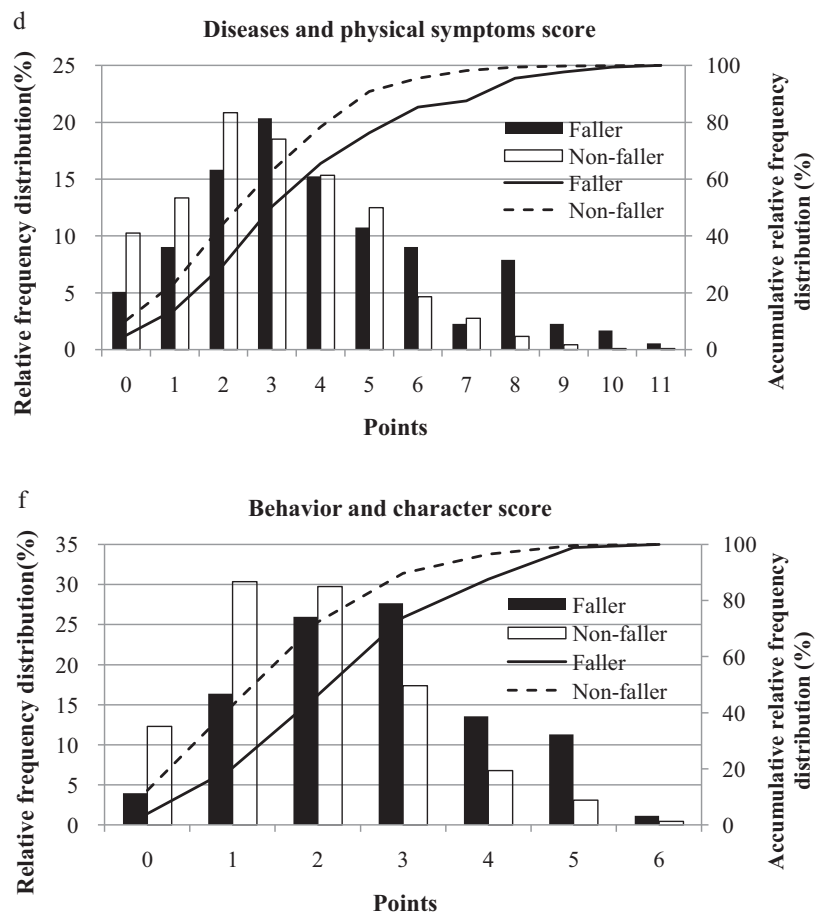

Fig. 1. The relative frequency distribution and accumulative relative frequency distribution in the total and every risk factor scores.

of distinguishing fallers and non-fallers correctly obtained from each discriminant analyses. The percentage of distinguishing fallers correctly when using the total score as an independent variable was $14.4 \%$, and that when using the five risk factor scores as independent variables was $39.7 \%$. Furthermore, these percentages when using each risk factor score as an independent variable were $42.5 \%$ (symptom of falling), $0.6 \%$ (physical function score),

Table 1

The summary of discriminant probabilities (\%).

\begin{tabular}{llrr}
\hline Independent variables & \multicolumn{3}{l}{$\begin{array}{l}\text { Percentages of distinguishing } \\
\text { correctly }\end{array}$} \\
\cline { 2 - 4 } & $\begin{array}{l}\text { Fallers and } \\
\text { non-fallers }\end{array}$ & Fallers & Non-fallers \\
& 85.3 & 14.4 & 98.5 \\
\hline Total score & 84.0 & 39.7 & 92.3 \\
5-Risk-factor scores & 82.0 & 42.5 & 89.4 \\
Symptoms of falling score & 84.2 & 0.6 & 99.8 \\
Physical function score & 84.4 & 0.6 & 100.0 \\
Disease and physical symptoms score & 84.3 & 0.0 & 100.0 \\
Environment score & 84.1 & 1.1 & 99.6 \\
Behavior and character score & 84.7 & 28.2 & 95.3 \\
TMIG score & 85.3 & 16.1 & 98.2 \\
TMIG score (excluding "fall & & & \\
\hline experience") & & & \\
\hline
\end{tabular}

0.6\% (disease and physical symptoms score), 0.0\% (environment score), and $1.1 \%$ (behavior and character score), respectively.

Furthermore, a similar analysis was conducted using the TMIG assessment chart. Because the TMIG assessment chart includes "fall experience" among its 15 assessment items, the total score was calculated by summing up scores of 14 items excluding the item of fall experience. As the result of discriminant analysis using fall experience as a dependent variable and the total score of TMIG as an independent variable, the percentage of fallers distinguished correctly was $16.1 \%$.

\subsection{Relationship between fall experience and total and risk factor scores of the DFRA}

To confirm the relationship between fall experience (fall risk) and the total and risk factor scores, odds ratio and incidence of fall experience were calculated for every point of the total or risk factor scores (Fig. 2a-f). The odds ratios calculated for every point of the total score ranged from 0.0 to 74.8 . Odds ratios were 0.0 when total score under 2 point, and were 5.0 or more at over 3 or 4 points of total score. Although odds ratios were under 5.0 when the total score ranged from 5 to 23 , they were 5.0 or more at 24 point or more of the total score. Incidence of fall showed increased 

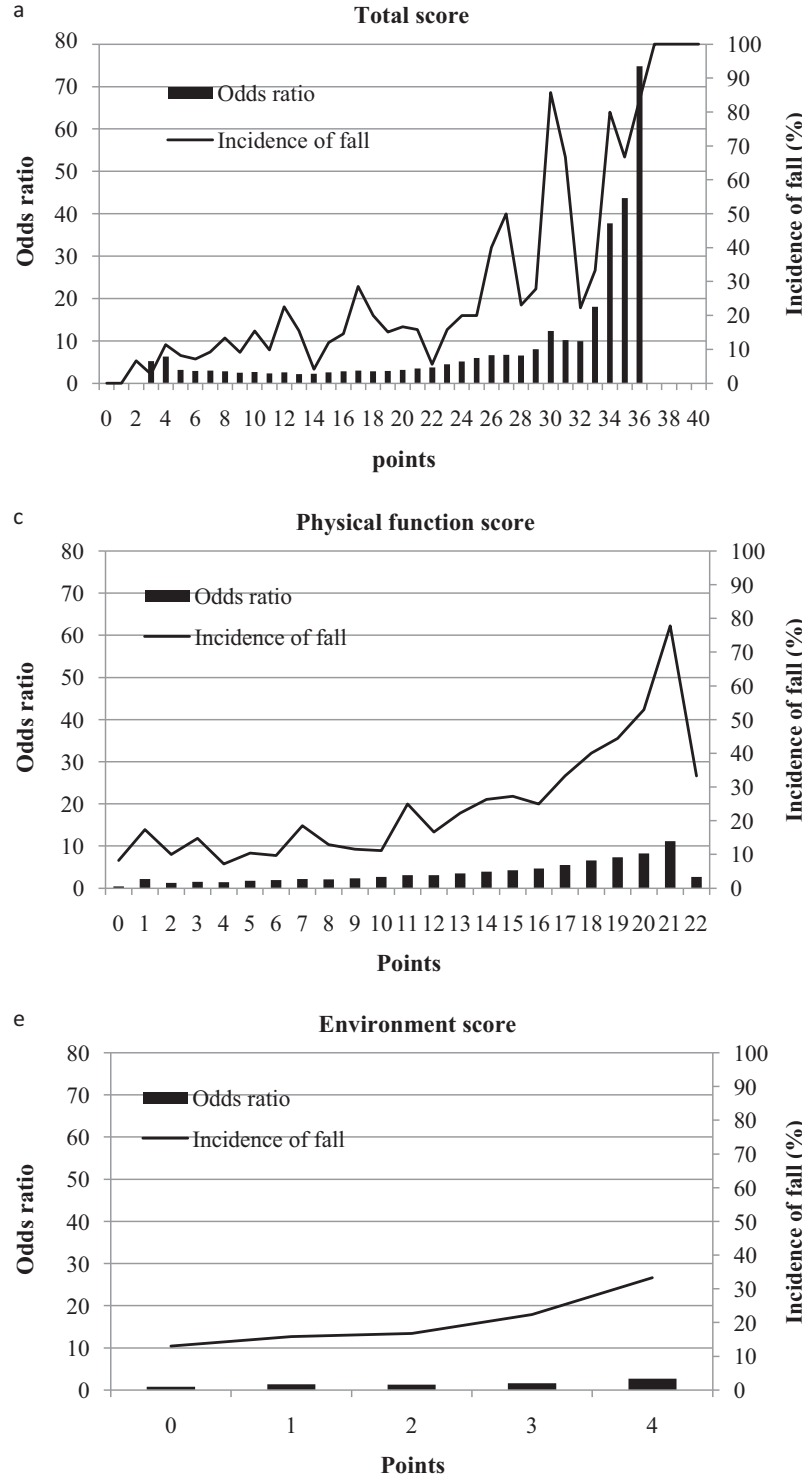
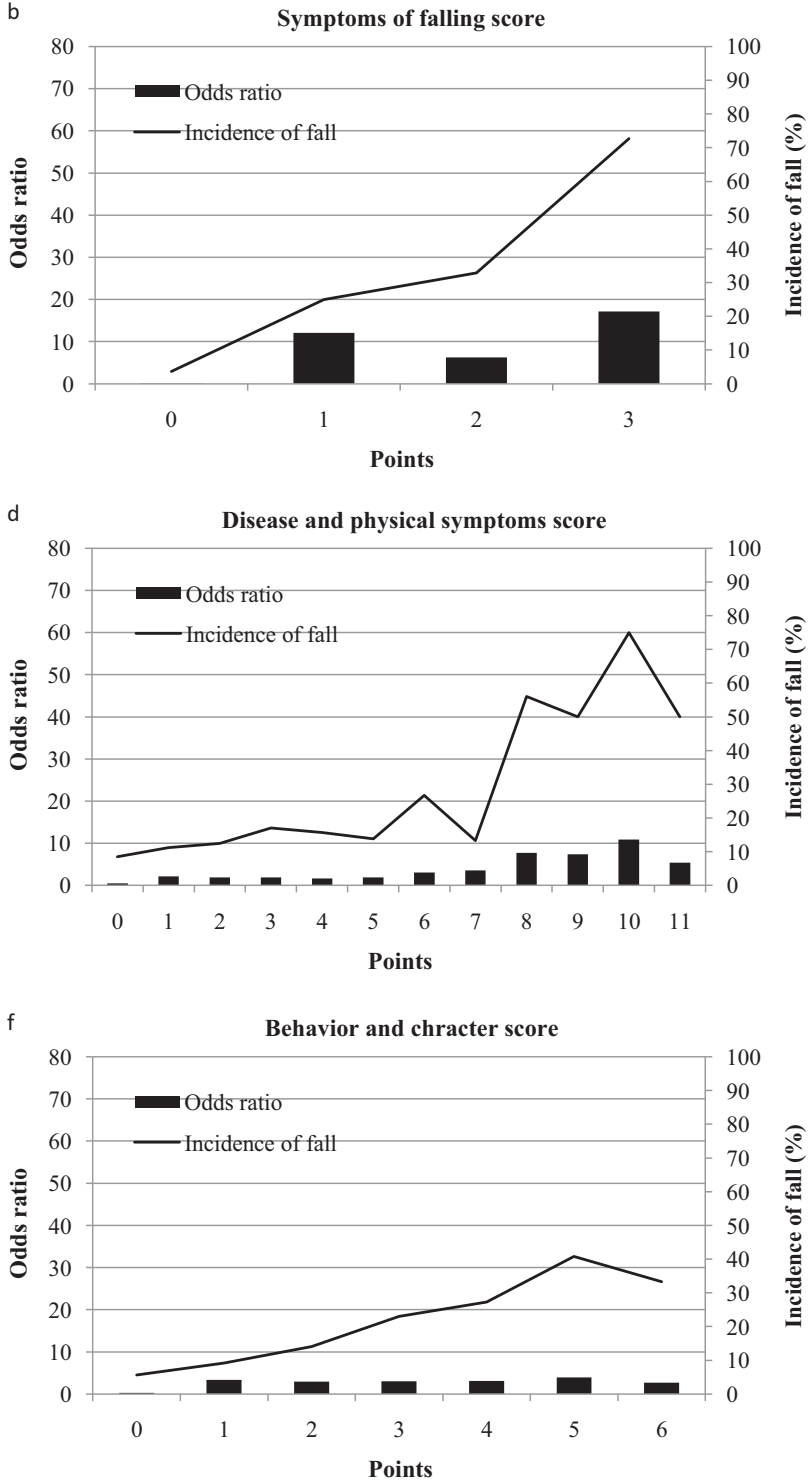

Fig. 2. The odds ratio and incidence of fall (\%) calculated for every point of the total and risk factor scores.

tendency with increasing total score, and reached $50 \%$ when the total score was 27 points.

Furthermore, we similarly examined each risk factor score. In all risk factor scores, odds ratios of 0 points of each risk factor score were under $1.0(0.0-0.7)$, and those at 1 point or more of each risk factor score were 1.0 or more. The ranges of odds ratios of each risk factor were 0.1-17.2 (symptom of falling), 0.5-11.1 (physical symptom), 0.5-10.9 (disease and physical symptoms), 0.7-2.7 (environment), 0.3-3.9 (behavior and character). On the other hand, points of each risk factor when the odds ratio reached 5.0 or more were 1 point (symptom of falling), 17 points (physical function), 8 points (disease and physical symptoms), 4 points (environment). The maximal odds ratio in "behavior and character" was 4.2 (5 points). In addition, incidence of fall in the each risk factors also showed increased tendency with increasing each risk factor score, and the highest values was $73 \%$ in the symptoms of falling ( 3 points), $78 \%$ in the physical function ( 21 points), $75 \%$ in the disease and physical symptoms (10 points), $33 \%$ in the environment ( 4 points), and $41 \%$ in the behavior and character ( 5 points).

\section{Discussion}

This study assumed that fall risk factors is composed of five factors of "symptoms of falling," "physical function," "disease and physical symptoms," "environment," and "behavior and character," and examined the validity of the DFRA. The accuracy of prediction of fall experience based on risk factor scores of the DFRA was about $40 \%$, and almost all of this could be explained by three items under "symptoms of falling." These results indicate the following suggestions about the accuracy of predicting fall experience.

First, a few items under the "symptoms of falling" work better for predicting fall experience (screening fall experience) in the healthy elderly population, compared to many items under other risk factors that were reported in previous studies. This was supported by the result that the percentage of predicting fall experience correctly from the TMIG score was low (16.1\%), though the TMIG assessment chart is composed of items regarding risk factors such as physical function, disease, environment, and fear of falling. Falling is a multifactoral problem (Graafmans et al., 1996 
Perell et al., 2001; Russell et al., 2009), and a cause of falling is highly individualized (Pluijm et al., 2006). Therefore, there may be a limitation in accurately predicting fall experience from the total score of relevant factors. Those items representing symptoms of falling in this study, such as "stumble," "felt like falling," and "about to fall," reflect the state of being liable to fall. The "symptom of falling" is an outcome reflecting complex risk factors as reported in many studies, and this factor may make it more possible to relate more directly to past or future falls over other risk factors. Several prospective studies have indicated that "currently falling" is an important predictor of "recurrently falling" (Pluijm et al., 2006). Furthermore, the reports screening fall risk from current falling and performance score are more common in the fall risk assessment based on the performance test (American Geriatrics Society, 2001; Perell et al., 2001; Pluijm et al., 2006; Laessoe et al., 2007; Muir et al., 2008; Russell et al., 2009). So, as for screening persons at high risk of falling, use of "symptoms of falling" (the state of being liable to fall) can provide more accurate and efficient assessment.

Secondly, it is important to not only assess risk level but also identify the risk profile for individuals, and these assessments are directly related to the prevention of falls in the future. In this study, significant differences in the total score and scores of each risk factor were found between faller and non-faller groups, and fall risk (odds ratios and incident of fall) tended to increase with the increase in the total score and each risk factor score. These suggest that the problems concerning these risk factors are somewhat connected to falls in the elderly. On the other hand, as mentioned above, it became clear that there is a limitation in the prediction of fall experience from questionnaire-based risk factor scores (or based on a questionnaire consisting of multiple risk factors). To directly relate outcomes of fall risk assessments to fall prevention, it is preferable that outcomes of fall risk assessment can provide not only comprehensive fall risk level but also identify personal problems for each risk factor (American Geriatrics Society, 2001). The TMIG chart has problems as follows: (1) there is a limitation in the assessment of a risk profile because this assessment chart is composed of multifactoral components, but is unbalanced; (2) there are many items regarding disease, which are difficult to improve in the short term, but there are a few items specifically regarding physical function. Considering the fact that fall prevention measurements for the healthy elderly population may be mainly focused on an improvement of physical function, comprehensive assessment of physical function characteristics is important to clarify physical problems for individuals. However, the TMIG fall risk assessment chart has only four items regarding walking ability, balancing, and muscular strength. In addition, since there are great individual differences of physical function level in the healthy elderly population, physical function should be only slightly assessed for each component. This is available to confirm effectiveness after fall prevention measurements.

This study assumed two or more sub-factors for each risk factor of "physical function," "disease and physical symptoms," "environment," and "behavior and character," respectively. Then, two or more assessment items were set for each sub-factor, and a comprehensive assessment of a risk profile is expected. Assessment items used in this study have the advantage of predicting physical function because of their comprehensive assessment of physical characteristics and a graduated assessment of physical function level. This advantage exists because of the physical function level assessment items in each sub-factor regarding physical function, such as balancing, muscular strength, lower limb strength, walking ability, going up and down stairs, changing and holding posture, and upper limb function.

Furthermore, this study gave meaning to the total score and each risk factor score by calculating the odds ratio and the incidence of fall experience for each score. As for the TMIG assessment chart, it may be possible to establish criteria for screening persons at high risk of falling by using the results of the odds ratio or the incidence of falling. However, considering the fact that the probability of distinguishing fall experience was low, illustrating the relationship between fall risk and each score may be applicable to fall prevention rather than establishing a standardized criterion for screening. In this study, it was impossible to provide an example of a risk profile and its practical use due to space limitations. This is a future issue.

This study was based on a cross-sectional data set, and had to use fall experience as a criterion for examining applicability of the total and risk factor scores. Since fall risk means the possibility of falling in the future, further examination using longitudinal data set will be required.

\section{Conflict of interest statement}

None.

\section{Acknowledgment}

This work was supported by A Grant-in-Aid for Science Research, the Japan Ministry of Education, Science, Sports and Culture [grant number 21240064].

\section{References}

American Geriatrics Society, British Geriatrics Society, and American Academy of Orthopaedic Surgeons Panel on falls prevention, 2001. Guideline for the prevention of falls in older persons. J. Am. Geriatr. Soc. 49, 664-672.

Chan, B.K.S., Marshall, L.M., Winters, K.M., Faulkner, K.A., Schwartz, A.V., Orwoll, E.S., 2006. Incident fall risk and physical activity and physical performance among older men. Am. J. Epidemiol. 165, 696-703.

Demura, S., Sato, S., Yokoya, T., Sato, T., 2010. Examination of useful items in the assessment of fall risk in the community-dwelling elderly Japanese population. Environ. Health. Prev. Med. 15, 169-179.

Graafmans, W.C., Ooms, M.E., Hofstee, H.M.A., Bezemer, P.D., Bouter, L.M., Lips, P. 1996. Falls in the elderly: a prospective study of risk factors and risk profiles. Am. J. Epidemiol. 143, 1129-1136.

Laessoe, U., Hoeck, H.C., Simonsen, O., Sinkjaer, T., Voigt, M., 2007. Fall risk in an active elderly 14 population - can it be assessed? J. Negat. Results Biomed. 26, 6-12.

Muir, S.W., Berg, K., Chesworth, B., Speechley, M., 2008. Use of the Berg balance scale for predicting multiple falls in community-dwelling elderly people: a prospective study. Phys. Ther. 88, 449-459.

Perell, K.L., Nelson, A., Goldman, R.L., Luther, S.L., Prieto-Lewis, N., Rubenstein, L.Z., 2001. Fall risk assessment measures: an analytic review. J. Gerontol. A: Biol. Sci. Med. Sci. 56, M761-M766.

Pluijm, S.M.F., Smit, J.H., Tromp, E.A.M., Stel, V.S., Deeg, D.J.H., Bouter, L.M., Lips, P., 2006. A risk profile for identifying community-dwelling elderly with a high risk of recurrent falling: results of a 3-year prospective study. Osteoporos. Int. 17, 417-425.

Russell, M.A., Hill, K.D., Day, L.M., Blackberry, I., Gurrin, L.C., Dharmage, S.C., 2009. Development of the falls risk for older people in the community (FROP-Com) screening tool. Age Ageing 38, 40-46.

Schenkman, M., Riegger-Krugh, C., 1997. Physical intervention for elderly patients with gait disorders. In: Masdeu, J.C., Sudarsky, L., Wolfson, L. (Eds.), Gait Disorders of Aging. Lippincott-Raven, Philadelphia, pp. 327-353.

Suzuki, T., 2000. Questionnaire for falls assessment of elderly people and its application. In: Health Assessment Manual. Kosei Kagaku Kenkyusho, Tokyo, (In Japanese), pp. 142-163.

Suzuki, T., 2003. Epidemiology and implications of falling among the elderly. Nippon Ronen Igakkai Zasshi 40, 85-94 (In Japanese).

Tinetti, M.E., Speechley, M., Ginter, S.F., 1988. Risk factors for falls among elderly persons living in the community. N. Engl. J. Med. 319, 1701-1707. 